\title{
On-line Chain Partitions of Up-growing Semi-orders
}

\author{
Stefan Felsner • Kamil Kloch • Grzegorz Matecki • Piotr Micek
}

Received: 24 September 2007 / Accepted: 25 July 2011 / Published online: 23 August 2011

(C) The Author(s) 2011. This article is published with open access at Springerlink.com

\begin{abstract}
On-line chain partition is a two-player game between Spoiler and Algorithm. Spoiler presents a partially ordered set, point by point. Algorithm assigns incoming points (immediately and irrevocably) to the chains which constitute a chain partition of the order. The value of the game for orders of width $w$ is a minimum number $\operatorname{val}(w)$ such that Algorithm has a strategy using at most val $(w)$ chains on orders of width at most $w$. We analyze the chain partition game for up-growing semi-orders. Surprisingly, the golden ratio comes into play and the value of the game is $\left\lfloor\frac{1+\sqrt{5}}{2} w\right\rfloor$.
\end{abstract}

Keywords On-line $\cdot$ Chain partition $\cdot$ Order $\cdot$ Dimension $\cdot$ Semi-order

\footnotetext{
S. Felsner

AG Diskrete Mathematik Institut für Mathematik, Technische Universität Berlin, Strasse des 17. Juni 136, 10623 Berlin, Germany e-mail: felsner@math.tu-berlin.de

URL: http://www.math.tu-berlin.de/ felsner/

K. Kloch

Embedded Systems Lab, University of Passau, Innstrasse 43, 94032 Passau, Germany

e-mail: kamil.kloch@uni-passau.de

URL: http://www.esl.fim.uni-passau.de/ kloch/
}

G. Matecki · P. Micek $(\bowtie)$

Theoretical Computer Science Department,

Jagiellonian University, Lojasiewicza 6, 30-348 Kraków, Poland

e-mail: Piotr.Micek@tcs.uj.edu.pl

URL: http://tcs.uj.edu.pl/Micek

G. Matecki

e-mail: Grzegorz.Matecki@tcs.uj.edu.pl

URL: http://tcs.uj.edu.pl/Matecki 


\section{Introduction}

On-line chain partitions of an order can be described as a two-person game between Algorithm and Spoiler. The game is played in rounds. Spoiler presents an on-line order, one point at a time. Algorithm responds by making an irrevocable assignment of the new point to one of the chains of the chain partition. The performance of Algorithm's strategy is measured by comparing the number of chains used with the number of chains of an optimal chain partition. By Dilworth's Theorem the size of an optimal chain partition equals the width of the order. The value of the game for orders of width $w$, denoted by $\operatorname{val}(w)$, is the least integer $n$ for which some Algorithm has a strategy using at most $n$ chains for every on-line order of width $w$. Alternatively, it is the largest integer $n$ for which Spoiler has a strategy that forces any Algorithm to use $n$ chains on order of width $w$.

The study of chain partition games goes back to the early 80's when Kierstead [4] (upper bound) and Szemerédi (lower bound published in [5]) proved the estimates for on-line orders of width $w:\left(\begin{array}{c}w+1 \\ 2\end{array}\right) \leqslant \operatorname{val}(w) \leqslant \frac{5^{w}-1}{4}$. It took almost 30 years until these bounds had been slightly improved. The story can be found in the survey [2].

The study of on-line chain partition on restricted classes of orders began in 1981 when Kierstead and Trotter [6] proved the following result: when Spoiler is restricted to presenting interval orders of width $w$, the value of the game is $3 w-2$. Among other classes of orders that have been studied thereafter are $(\mathbf{k}+\mathbf{k})$-free orders and semi-orders. Again we refer to [2] for details.

Up-growing on-line orders have been introduced by Felsner [3]. In this variant Spoiler's power is restricted by the condition that the new element has to be a maximal element of the order presented so far. Felsner [3] showed that the value of the chain partition game on up-growing orders is $\left(\begin{array}{c}w+1 \\ 2\end{array}\right)$. The case of up-growing interval orders was resolved by Baier et al. [1]. The value of the game in this variant is $2 w-1$.

This paper resolves the on-line chain partition problem for up-growing semiorders. An order $\mathbf{P}$ is called a semi-order if it has a unit interval representation, i.e., there exists a mapping $I$ of points of the order into unit length intervals on a real line so that $x<y$ in $\mathbf{P}$ iff interval $I(x)$ is entirely to the left of $I(y)$. Alternatively semi-orders are characterized as the $(\mathbf{2}+\mathbf{2})$ and $(\mathbf{3}+\mathbf{1})$-free orders (see Fig. 2$)$.

Considering on-line chain partitions of semi-orders note that the general (not upgrowing) case is easy to analyze: First, observe that the number of chains used by Algorithm can be bounded by $2 w-1$. Let $x$ be the new point and consider the set $\operatorname{Inc}(x)$ of points incomparable with $x$. Clearly, the only chains forbidden for $x$ are those used in $\operatorname{Inc}(x)$. Now width $(\operatorname{Inc}(x)) \leqslant w-1$ since the width of the whole order does not exceed $w$. Moreover, height $(\operatorname{Inc}(x)) \leqslant 2$ as the presented order is $(\mathbf{3}+\mathbf{1})$ free. Therefore, $|\operatorname{Inc}(x)| \leqslant 2(w-1)=2 w-2$, proving that $x$ can be assigned to at least one of $2 w-1$ legal chains.

It turns out that there is no better strategy for Algorithm. In other words, Spoiler may force Algorithm to use $2 w-1$ chains on semi-orders of width $w$. A strategy for Spoiler looks as follows:

(1) Present two antichains $A$ and $B$, both consisting of $w$ points in such a way that $A<B$, i.e., all points from $A$ are below all points from $B$. If Algorithm uses $2 w-1$ or more chains, the construction is finished. Otherwise, suppose that $k$ 
Fig. 1 Strategy for Spoiler forcing Algorithm to use $2 w-1$ chains

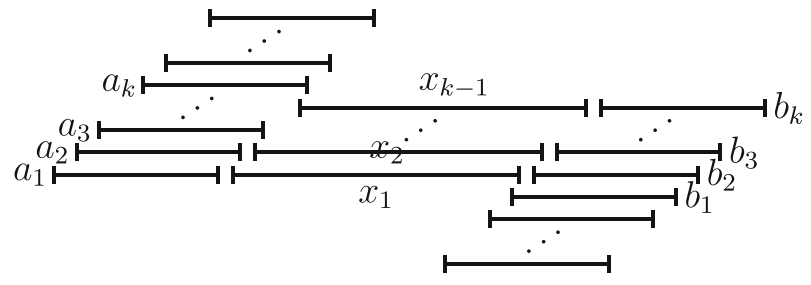

chains $(2 \leqslant k \leqslant w)$ contain elements from $A$ and $B$, namely let $a_{i} \in A_{i}, b_{i} \in B_{i}$ for $1 \leqslant i \leqslant k$ lie in the same chain.

(2) Present $k-1$ points $x_{1}, \ldots, x_{k-1}$ in such a way that $\left\{a_{1}, \ldots, a_{i}\right\} \leqslant x_{i} \leqslant$ $\left\{b_{i+1}, \ldots, b_{k}\right\}$ and $x_{i}$ is incomparable to all the rest (the interval representation of the whole order looks as in Fig. 1). It is easy to verify that in such setting Algorithm is forced to use $2 w-1$ chains.

The contribution of this paper is the following theorem.

Theorem 1.1 The value of the on-line chain partition game for up-growing semiorders of width $w$ is $\left\lfloor\frac{1+\sqrt{5}}{2} \cdot w\right\rfloor$.

\section{Up-growing Semi-orders}

\subsection{Outline}

In this section we prove that the value of the on-line chain partition game for upgrowing semi-orders equals $\lfloor\varphi \cdot w\rfloor$, where $\varphi=\frac{1+\sqrt{5}}{2}$ is the golden number. First, in Section 2.2 we collect some facts about semi-orders. Section 2.3 describes a strategy for Spoiler which forces Algorithm to use at least $\lfloor\varphi \cdot w\rfloor$ chains on a semi-order of width $w$. This sets the lower bound for the value of the game. In Section 2.4 we propose a strategy for Algorithm using at most $\lfloor\varphi \cdot w\rfloor$ chains on semi-orders of width at most $w$.

The presence of the golden number $\varphi$ in the result of a chain partition game may seem surprising. In fact, it is the Fibonacci sequence $\left(F_{0}=0, F_{1}=1\right.$ and $F_{i+2}=F_{i}+$ $\left.F_{i+1}\right)$ which appears in the counting argument of the upper bound and serves as a discrete counterpart of $\varphi$.

\subsection{Basic Facts}

For $x, y \in \mathbf{P}$ by $x \|_{\mathbf{P}} y$ we mean that $x$ and $y$ are incomparable in $\mathbf{P}$. If $x$ is grater that $y$ (or, $x$ is grater that each point from a set $Y$ ) we write shortly $x$ dominates $y$ (or, $x$ dominates $Y$ ). Let $\mathrm{D}_{\mathbf{P}}(x)=\{y \in P: y<x\}$, called a down set of $x$ in $\mathbf{P}$, denote the set of predecessors of $x$ in P. Dually, let $\mathrm{U}_{\mathbf{P}}(x)=\{y \in P: y>x\}$, called an up set of $x$, denote the set of successors of $x$ in $\mathbf{P}$. If the order $\mathbf{P}$ is unambiguous from the context we also write $\mathrm{U}(x)$ instead of $\mathrm{U}_{\mathbf{P}}(x)$ and $\mathrm{D}(x)$ instead of $\mathrm{D}_{\mathbf{P}}(x)$. $\mathrm{By} \mathrm{D}(X)$ we mean $\bigcup_{x \in X} \mathrm{D}(x)$.

The maximum and the minimum elements of a chain $\gamma$ are denoted respectively by $\operatorname{top}(\gamma)$ and $\operatorname{bottom}(\gamma)$. 
Fig. $2(\mathbf{2}+\mathbf{2})$ and $(\mathbf{3}+\mathbf{1})$

orders

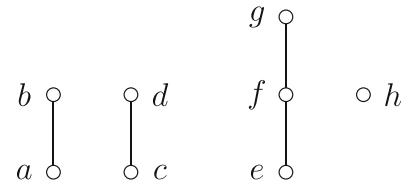

An order $\mathbf{P}$ is called an interval order if it has an interval representation, i.e., there exists a mapping $I$ of points of the order into intervals on a real line so that $x<y$ in $\mathbf{P}$ iff $\max I(x)<\min I(y)$. Interval orders have several nice characterizations, see e.g. [7]. In our context the following two will be used repeatedly:

(1) $\mathbf{P}=(P, \leqslant)$ is an interval order iff the set of down sets (up sets) of elements of $\mathbf{P}$ is linearly ordered with respect to inclusion, i.e., for $p, q \in P$ either $\mathrm{D}(p) \subseteq \mathrm{D}(q)$ or $\mathrm{D}(p) \supseteq \mathrm{D}(q)$. Note that this ordering of down sets corresponds to the order of left endpoints in an interval representation.

(2) $\mathbf{P}=(P, \leqslant)$ is an interval order iff $\mathbf{P}$ is a $(\mathbf{2}+\mathbf{2})$-free order, i.e., $\mathbf{P}$ does not contain elements $a, b, c, d \in P$ such that: $a<b, c<d, a \| d$ and $c \| b$ (see Fig. 2).

An interval order $\mathbf{P}$ is called a semi-order if it has a unit interval representation, i.e., all intervals are of the same length. Semi-orders are also characterized in terms of forbidden structures: an interval order $\mathbf{P}$ is a semi-order iff $\mathbf{P}$ is a $(\mathbf{3}+\mathbf{1})$-free order, i.e., $\mathbf{P}$ does not contain elements $e, f, g, h \in P$ such that $e<f<g$ and $h \| e, f, g$ (see Fig. 2).

\subsection{The Lower Bound}

Fix $w$ and consider the system $\left(I_{k}\right)$ of $k+1$ linear inequalities

$$
x_{0}+x_{1}+\ldots+x_{j-1}+2 x_{j}-x_{j+1} \leqslant w, j=0, \ldots, k .
$$

From the following two propositions it immediately follows that there exists a strategy for Spoiler which forces Algorithm to use $\lfloor\varphi \cdot w\rfloor$ chains on an up-growing semi-order of width $w$. This is the lower bound needed for Theorem 1.1.

Proposition 2.1 If $\left(x_{0}, x_{1}, \ldots, x_{k}, x_{k+1}\right)$ is an integral solution of $\left(I_{k}\right)$ with $x_{0} \geqslant x_{1} \geqslant$ $\ldots \geqslant x_{k} \geqslant x_{k+1}=0$ then there is a strategy for Spoiler to present an up-growing semiorder of width $w$ and force Algorithm to use at least $w+x_{0}$ chains.

Proposition 2.2 For each $w$ there is an integer $k$ and an integral solution of $\left(I_{k}\right)$ with $x_{0}=\lfloor(\varphi-1) \cdot w\rfloor \geqslant x_{1} \geqslant \ldots \geqslant x_{k}>x_{k+1}=0$.

Proof of Proposition 2.1 Fix $w, k>0$ and an integer solution $\left(x_{0}, \ldots, x_{k}\right)$ of $\left(I_{k}\right)$ with $x_{0} \geqslant x_{1} \geqslant \ldots \geqslant x_{k} \geqslant x_{k+1}=0$. The strategy for Spoiler induced by $\left(x_{0}, \ldots, x_{k}\right)$ presents an up-growing semi-order $\mathbf{P}=(P, \leqslant)$ of width $w$. The points of $\mathbf{P}$ are presented in bundles so that the actual presentation sequence has the following structure

$$
P=\left(A, C_{0}, B_{1}, C_{1}, B_{2}, C_{2}, \ldots, C_{k}, B_{k+1}\right) .
$$


The height of $\mathbf{P}$ is at most 3 where $A$ is the set of all minimal elements, points of height 2 lie in $C_{0} \cup \bigcup_{i=1}^{k+1} B_{i}$ and all points from $\bigcup_{i=1}^{k} C_{i}$ are of height 3. In particular, the down sets of points in $\bigcup B_{j}$ are subsets of $A$, and the down sets of points in $\bigcup C_{j}$ are the union of $A$ and a subset of $\bigcup B_{j}$.

Throughout the construction Spoiler maintains auxiliary sets $D_{1}, \ldots, D_{k}$. Initially $D_{i}=\varnothing$ for every $i$. During the construction the following invariant will be kept:

$$
D_{i} \subseteq B_{i} \text { and } D_{i} \text { does not contain top of any chain used by Algorithm. }
$$

Now, we describe the phases of the construction. The proof that the construction has all desired properties will follow thereafter.

Spoiler starts the construction by presenting an antichain $A$ of size $w$. Algorithm has to use $w$ different chains.

Phase $j(0 \leqslant j \leqslant k)$. In the $j$-th phase Spoiler builds $x_{j}-x_{j+1}$ forcing paths. The points constituting these paths will go into the set $C_{j}$.

The first point $q_{0}$ of a forcing path dominates $A \cup \cup_{i \leqslant j} B_{i}$. Now, suppose that the first $i+1$ points of the path have been presented and let $q_{i}$ be the last of these points. If Algorithm assigned $q_{i}$ to a new chain or to a chain whose top is in $A$, then $q_{i}$ is the last point of the forcing path. Otherwise, $q_{i}$ was assigned to some chain with a top $b \in B_{s}$. In this case Spoiler updates $D_{s}:=D_{s} \cup\{b\}$ and then introduces $q_{i+1}>A \cup B_{1} \cup \ldots \cup B_{s-1} \cup D_{s}$.

Note that the invariant (1) is kept, i.e., $D_{i}$ does not contain any chain top. Algorithm has to assign $q_{i+1}$ to a new chain or to a chain with top in $A \cup B_{1} \cup \ldots \cup B_{S-1}$. This means that if $q_{i+1}$ is assigned to a chain with a top from $B_{s^{\prime}}$ then $s^{\prime}<s$. Thus, consecutive points $q_{0}, q_{1}, \ldots$ of a forcing path (excluding the last one) are assigned to chains with tops from the $B_{i}$ 's with decreasing indices. This proves that the path is finite.

The intuition is that with each forcing path Spoiler forces Algorithm to produce a skip chain, i.e., a chain of height 2 with its bottom in $A$ and its top in $C_{j}$ (avoiding all the $B_{i}$ 's), or to use a brand new chain.

Assume that Spoiler constructed all the forcing paths and consider the set $A_{j} \subseteq$ $A$ of bottom points of skip chains with tops in $C_{j}$. Clearly, $\left|A_{j}\right| \leqslant x_{j}-x_{j+1}$. Now, Spoiler introduces a set $B_{j+1}$ of size $x_{j}-x_{j+1}$ such that each point $p \in B_{j+1}$ dominates $A_{j} \cup \mathrm{D}\left(B_{j}\right)$ and $|\mathrm{D}(p)|=\left|\mathrm{D}\left(B_{j+1}\right)\right|=x_{0}-x_{j+1}$ (put $B_{0}=\emptyset$ ). This means that if $\left|A_{j}\right|<x_{j}-x_{j+1}$ or $A_{j} \cap \mathrm{D}\left(B_{j}\right) \neq \emptyset$ then Spoiler completes $\mathrm{D}\left(B_{j+1}\right)$ with arbitrarily chosen points from $A-\left(A_{j} \cup \mathrm{D}\left(B_{j}\right)\right)$. This is possible as $\left|\mathrm{D}\left(B_{j}\right)\right|+\left|A_{j}\right| \leqslant\left(x_{0}-x_{j}\right)+$ $\left(x_{j}-x_{j+1}\right)=x_{0}-x_{j+1} \leqslant w=|A|$, by $\left(I_{k}\right)$.

To prove that the construction actually works and thus concludes the proof of Proposition 2.1 we have to verify the following three facts.

Fact 2.3 P is a semi-order.

Fact 2.4 The width of $\mathbf{P}$ is $w$.

Fact 2.5 Algorithm has to use at least $w+x_{0}$ chains to cover $\mathbf{P}$.

Proof of Fact 2.3 We proceed in two steps, first we show that $\mathbf{P}$ is an interval order and then that it is $(\mathbf{3}+\mathbf{1})$-free. 
In order to prove that $\mathbf{P}$ is an interval order we show that the down sets of points from $\mathbf{P}$ are linearly ordered with respect to inclusion. Indeed,

(i) $\mathrm{D}(A)=\emptyset$,

(ii) $\mathrm{D}\left(B_{1}\right) \subseteq \mathrm{D}\left(B_{2}\right) \subseteq \ldots \subseteq \mathrm{D}\left(B_{k+1}\right) \subseteq A$,

(iii) $\mathrm{D}\left(C_{0}\right)=A$,

(iv) if $c \in C_{j}$ is the starting point of a forcing path, then $\mathrm{D}(c)=A \cup\left(B_{1} \cup \ldots \cup B_{j}\right)$,

(v) if $c \in C_{j}$ is not a starting point, then there is an $s$ with $\mathrm{D}(c)=A \cup\left(B_{1} \cup \ldots \cup\right.$ $\left.B_{s-1} \cup D_{s}^{t}\right)$.

The set $D_{s}^{t}$ from the last line refers to the respective set $D_{s}$ at the moment when $c$ is introduced. Recalling that $D_{s}$ can only grow over time and $D_{s} \subseteq B_{s}$ we can conclude that the down sets of elements of $c$ are linearly ordered with respect to inclusion. Hence $\mathbf{P}$ is an interval order.

To see that $\mathbf{P}$ is a semi-order suppose that $\mathbf{P}$ contains a $(\mathbf{3}+\mathbf{1})$-configuration $d \|$ $a, b, c$ with $a<b<c$. Since $\mathbf{P}$ has height at most 3 and $A<\bigcup_{i=0}^{k} C_{i}$, the only option is that $a \in A, b, d \in \bigcup_{i=1}^{k+1} B_{i}$ and $c \in \bigcup_{i=1}^{k} C_{i}\left(C_{0}\right.$ is excluded as it is incomparable to the $B_{i}$ 's). Let $i, j$ be such that $b \in B_{i}$ and $d \in B_{j}$. Then it is easy to see that $a<d$ if $i \leqslant j$ (as in this case $a \in \mathrm{D}(b) \subseteq \mathrm{D}(d)$ ) and $d<c$ otherwise (as $c$ being an element of a forcing path with $c>b \in B_{i}$ implies $c>B_{1} \cup \ldots \cup B_{i-1} \supseteq B_{j}$ ). This contradiction to $d \| a, c$ shows that $\mathbf{P}$ is $(\mathbf{3}+\mathbf{1})$-free so it is a semi-order.

Proof of Fact 2.4 To prove that width $(\mathbf{P})=w$ consider any antichain $X$ in $\mathbf{P}$. We will show that $|X| \leqslant w$. Let $m \in X$ be the point with a maximal down set among points in $X$. We distinguish between three cases:

- If $m \in A$, then $X \subseteq A$ and $|X| \leqslant|A|=w$.

- If $m \in B_{i}$, then $X \subseteq B_{1} \cup \ldots \cup B_{i} \cup\left(A-\mathrm{D}\left(B_{i}\right)\right)$ and $|X| \leqslant\left(x_{0}-x_{1}\right)+\ldots+\left(x_{i-1}-\right.$ $\left.x_{i}\right)+\left(w-\left(x_{0}-x_{i}\right)\right)=w$.

- If $m \in C_{0}$, then $X \subseteq \bigcup_{i} B_{i}$ and $|X| \leqslant\left|C_{0}\right|+\left|B_{1}\right|+\ldots+\left|B_{k+1}\right|=\left(x_{0}-x_{1}\right)+$ $\left(x_{0}-x_{1}\right)+\ldots+\left(x_{k}-x_{k+1}\right)=x_{0}+x_{0}-x_{1} \leqslant w$ by $\left(I_{k}\right)$.

The last and most interesting case is when $m \in C_{j}$ for some $j>0$. We may write $\mathrm{D}(m)=A \cup\left(B_{1} \cup \ldots \cup B_{s-1} \cup D_{s}^{t}\right)$ for $1 \leqslant s \leqslant j+1$ where again $D_{s}^{t}$ is the set $D_{s}$ at the moment when $m$ was inserted. When $m$ is the starting point of a forcing path we have $s=j+1$ and $D_{j+1}^{t}=\emptyset$, since $D_{j+1}^{t} \subseteq B_{j+1}$ and $B_{j+1}$ is presented after $C_{j}$. Clearly, $X \subseteq Y \cup\left(\bigcup_{i} B_{i}-\mathrm{D}(m)\right)$ where $Y=\left\{c \in \bigcup_{i} C_{i}: \mathrm{D}(c) \subseteq \mathrm{D}(m)\right\}$.

Since the starting points of forcing paths in $Y$ were introduced in phases 0 to $s-1$, their total number is $\left(x_{0}-x_{1}\right)+\left(x_{1}-x_{2}\right)+\ldots+\left(x_{s-1}-x_{s}\right)=x_{0}-x_{s}$. The introduction of each $c \in Y$ being not a starting point of a forcing path is preceded by an extension of some $D_{i}$ for $1 \leqslant i \leqslant s$. Therefore the number of non-starting points in $Y$ is bounded by $\left|D_{1}\right|+\ldots+\left|D_{s-1}\right|+\left|D_{s}^{t}\right|$. To simplify this expression we first prove the following bound:

$$
\left|D_{i}\right| \leqslant x_{i}
$$

For the proof of the inequality note that the set $D_{i}$ is enlarged only if a point from a forcing path is assigned to a chain with a top from $B_{i}$. This can only happen for forcing paths presented in phases following phase $i$. Each forcing path can contribute at most one point to $D_{i}$. There are $\left(x_{i}-x_{i+1}\right)+\ldots+\left(x_{k}-x_{k+1}\right)$ forcing paths in phases presented after phase $i$. Since $x_{k+1}=0$ this is not greater than $x_{i}$, as claimed. 
Collecting pieces from above we get $|Y| \leqslant\left(x_{0}-x_{s}\right)+\left|D_{1}\right|+\ldots+\left|D_{s-1}\right|+\left|D_{s}^{t}\right| \leqslant$ $\left(x_{0}-x_{s}\right)+x_{1}+\ldots+x_{s-1}+\left|D_{s}^{t}\right|$.

Recall that $\bigcup_{i} B_{i}-\mathrm{D}(m)=\left(B_{s}-D_{s}^{t}\right) \cup B_{s+1} \cup \ldots B_{k+1}$. All this finally yields:

$$
\begin{aligned}
|X| \leqslant & |Y|+\left|\bigcup_{i} B_{i}-\mathrm{D}(m)\right| \\
= & {\left[\left(x_{0}-x_{s}\right)+x_{1}+\ldots+x_{s-1}+\left|D_{s}^{t}\right|\right] } \\
& +\left[\left(x_{s-1}-x_{s}-\left|D_{s}^{t}\right|\right)+\left(x_{s}-x_{s+1}\right)+\ldots+\left(x_{k}-x_{k+1}\right)\right] \\
= & x_{0}+x_{1}+\ldots+x_{s-1}+\left(x_{s-1}-x_{s}\right)
\end{aligned}
$$

From $\left(I_{k}\right)$ we know that this last expression is not greater than $w$.

Proof of Fact 2.5 We will prove that Algorithm is forced to use at least $w+x_{0}$ chains on $\mathbf{P}$. First, we show that

all points in $A-\mathrm{D}\left(B_{k+1}\right)$ are tops of the chains to which they are assigned.

For the proof of this statement first consider points in $\mathbf{P}$ dominating $A-\mathrm{D}\left(B_{k+1}\right)$. These are exactly the points in $\bigcup_{i} C_{i}$. Recall that if Algorithm produced a skip chain and assigned $c \in \bigcup C_{i}$ to a chain whose top was equal to $a \in A$ then $c$ ends a forcing path. If this forcing path was played in phase $j$, then Spoiler later presented $B_{j+1}$ in such a way that $B_{j+1}>a$ and therefore $a \in \mathrm{D}\left(B_{j+1}\right) \subseteq \mathrm{D}\left(B_{k+1}\right)$ so $a \notin A-\mathrm{D}\left(B_{k+1}\right)$ and we are done.

Consider the set $E$ of end points of forcing paths presented in the game. The key fact is that all points in $\left(A-\mathrm{D}\left(B_{k+1}\right)\right) \cup \bigcup_{i} B_{i} \cup E$ are covered with distinct chains. Indeed, we have shown that chains in $A-\mathrm{D}\left(B_{k+1}\right)$ are tops of the chains. End points of forcing paths are, by definition, in a chain that is not used in $\bigcup_{i} B_{i}$. Recall that

(i) $\left|A-\mathrm{D}\left(B_{k+1}\right)\right|=w-x_{0}$,

(ii) $\left|B_{1}\right|+\ldots+\left|B_{k+1}\right|=\left(x_{0}-x_{1}\right)+\ldots+\left(x_{k}-x_{k+1}\right)=x_{0}$,

(iii) $|E|=\left(x_{0}-x_{1}\right)+\left(x_{1}-x_{2}\right)+\ldots+\left(x_{k}-x_{k+1}\right)=x_{0}$.

Hence $\left|A-\mathrm{D}\left(B_{k+1}\right)\right|+\sum_{i}\left|B_{i}\right|+|E|=w+x_{0}$ which gives the lower bound on the number of chains used by Algorithm.

Proof of Proposition 2.2 We will show that for any $w$ there is a solution of $\left(I_{k}\right)$ with $x_{0}=\lfloor(\varphi-1) \cdot w\rfloor$. Consider the following sequence:

$$
\begin{aligned}
x_{0} & =\lfloor(\varphi-1) \cdot w\rfloor, \\
x_{j+1} & =\left\lfloor(\varphi-1) \cdot\left(w-x_{0}-\ldots-x_{j}\right)\right\rfloor .
\end{aligned}
$$

Note that for any $0 \leqslant a \leqslant x$ we have $\lfloor(\varphi-1)(x-a)\rfloor \leqslant(\varphi-1) x-(\varphi-1) a<(\varphi-1) x$ and thus $\lfloor(\varphi-1)(x-a)\rfloor \leqslant\lfloor(\varphi-1) x\rfloor$. It implies that the sequence of $x_{j}$ 's is decreasing. Moreover, it eventually gets to zero since the partial sum $x_{0}+\ldots+x_{j}$ is getting larger. In particular there is a $k$ such that $x_{0} \geqslant \ldots \geqslant x_{k}>x_{k+1}=0$. It is easy to verify that the sequence is a solution of $\left(I_{k}\right)$, indeed

$$
x_{j} \leqslant(\varphi-1)\left(w-x_{0}-\ldots-x_{j-1}\right)=\frac{\varphi}{\varphi+1}\left(w-x_{0}-\ldots-x_{j-1}\right) .
$$


Fig. 3 Order $\mathbf{P}$ with its unit interval representation and the chain partition $\Gamma$ of points $a, b, c, d$

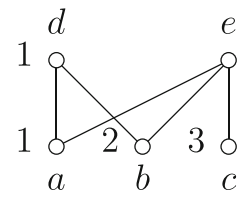

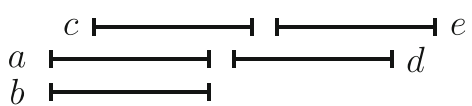

Multiplying this by $\varphi+1$, moving the term $\varphi x_{j}$ to the right hand side, adding $x_{0}+$ $\ldots+x_{j}$ on both sides and $-w+w$ on the right side we get

$$
x_{0}+\ldots+x_{j-1}+2 x_{j} \leqslant(\varphi-1)\left(w-x_{0}-\ldots-x_{j}\right)+w .
$$

The left side is an integer, therefore we may take the floor of the right side without affecting the truth. This results in an inequality from the system $\left(I_{k}\right)$ :

$$
x_{0}+\ldots+x_{j-1}+2 x_{j} \leqslant\left\lfloor(\varphi-1)\left(w-x_{0}-\ldots-x_{j}\right)\right\rfloor+w=x_{j+1}+w .
$$

Hence $\left(x_{0}, \ldots, x_{k}\right)$ is indeed a solution of $\left(I_{k}\right)$, concluding the proof of Proposition 2.2

\subsection{The Upper Bound}

Consider a semi-order $\mathbf{P}=(P, \leqslant)$ with $P=(a, b, c, d, e)$ and the chain partition $\Gamma: P \backslash\{e\} \rightarrow \mathbb{N}$ as shown in Fig. 3. Point $e$ may be covered with a new chain (say, with number 4) or with one of the chains already used. In the latter case Algorithm may choose between 2 and 3. We say that chain $\alpha$ is valid for a new point $x$ extending an already partitioned order $\mathbf{P}$ if $x$ dominates all points from $\alpha$ in $\mathbf{P}$. We claim that among the valid chains 2 and 3 defining $\Gamma(e)=3$ is the better choice. Indeed, any future point $p$ presented by Spoiler and dominating $c$ will also dominate $b$ (otherwise, $\mathbf{P}$ would have a $(\mathbf{2}+\mathbf{2})$ configuration which is forbidden in interval orders). On the other hand, Spoiler may play $q$ greater than $b$ but remaining incomparable to $c$ (see Fig. 4). Hence, using the chain of $c$ for $e$ leaves more options for the future. Whenever the chains of two points $x$ and $y$ are valid and $\mathrm{U}(x) \subsetneq \mathrm{U}(y)$ then it seems safer to use the chain of $x$. Our Algorithm ALG will go along this intuition.

Suppose that Spoiler introduces a semi-order $\mathbf{P}=(P, \leqslant)$ with a presentation order $P=\left(p_{1}, \ldots, p_{n}\right)$. We refer to the chains used by ALG as ALG-chains or just chains. With $\mathbf{P}_{x}$ we denote the order consisting of all points presented prior to $x$. We say that a chain $\alpha$ is valid for point $x$ if $\operatorname{top}(\alpha)$ in $\mathbf{P}_{x}$ is below $x$ in $\mathbf{P}$. The options of ALG are to put $x$ into some valid chain or into a new chain. We are ready to describe Algorithm's strategy. Let $x$ be a new point presented by Spoiler.

Fig. 4 Point $q$ may be presented by Spoiler in the future, point $p$ can not
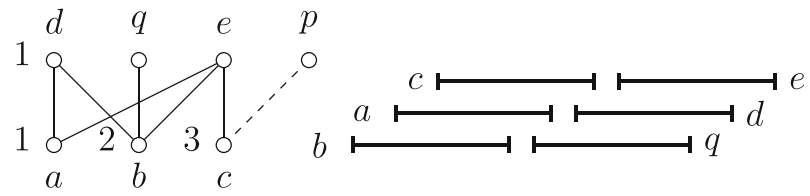
Algorithm ALG If there is a valid chain for $x$, then put $x$ into a valid chain $\alpha$ such that $\mathrm{U}(\operatorname{top}(\alpha)) \subseteq \mathrm{U}(\operatorname{top}(\beta))$ in $\mathbf{P}_{x}$ for all valid chains $\beta$. Otherwise, if there is no valid chain, use a new chain for $x$.

ALG is a greedy algorithm, i.e., it uses a new chain only when it is left with no other option. Note also that ALG has some freedom in choosing a chain for a new point $x$ as there may be many tops of valid chains for $x$ with the same minimum up set in $\mathbf{P}_{x}$.

The bound on the performance of ALG on width $w$ is stated in the following proposition. This is the upper bound part for Theorem 1.1.

Proposition 2.6 ALG uses at most $\varphi \cdot w$ chains on any up-growing semi-order of width at most $w$.

Suppose Spoiler presents a semi-order $\mathbf{P}=(P, \leqslant)$ with the presentation order $P=\left(p_{1}, \ldots, p_{n}\right)$ and width $(\mathbf{P}) \leqslant w$. We may assume that ALG uses a new chain for the last point $p_{n}$. If this were not the case Spoiler could stop the game earlier and the number of chains used by ALG would remain the same.

We partition $\mathbf{P}$ into layers. These layers will in some way reflect the preferences of ALG during the game. The point $p \in \mathbf{P}$ is significant if $p$ dominates at least one maximal point of $\mathbf{P}_{p}$. By the linearity of down sets of an interval order $\mathbf{P}$ this is equivalent to the condition that $p$ has the largest down set at the moment of its presentation. Let $e_{1}, \ldots, e_{m-1}$ be the significant points of $\mathbf{P}$, sorted with respect to the presentation order (If $\mathbf{P}$ has no significant points then $\mathbf{P}$ is an antichain and the thesis is trivial). These points define the partition of $\mathbf{P}$ into layers as follows (put $\left.\mathrm{D}\left(e_{0}\right)=\emptyset\right)$ :

$$
\begin{aligned}
D_{i} & =\mathrm{D}\left(e_{i}\right)-\mathrm{D}\left(e_{i-1}\right), \quad \text { for } 1 \leqslant i<m, \\
D_{m} & =P-\mathrm{D}\left(e_{m-1}\right) .
\end{aligned}
$$

Thus, $D_{i}$ (for $1 \leqslant i<m$ ) is exactly the set of maximal points of $\mathbf{P}_{e_{i}}$ covered by $e_{i}$. Here is a list of helpful and easy properties of the $D_{i}$ 's.

\section{Fact 2.7}

(i) For each $d_{1}, \ldots, d_{m}$ with $d_{i} \in D_{i}, \mathrm{U}\left(d_{1}\right) \supsetneq \mathrm{U}\left(d_{2}\right) \supsetneq \ldots \supsetneq \mathrm{U}\left(d_{m}\right)$.

(ii) $D_{m}$ is exactly the set of maximal points of $\mathbf{P}$. In particular, $p_{n} \in D_{m}$.

(iii) $D_{i}$ is an antichain, for every $i$.

(iv) If $d_{i} \in D_{i}, p \in P$ and $d_{i}<p$ then $D_{1} \cup \ldots \cup D_{i-1} \subseteq \mathrm{D}(p)$.

(v) If $d_{i} \in D_{i}, p \in P$ and $d_{i} \nless p$ then $\mathrm{D}(p) \subseteq D_{1} \cup \ldots \cup D_{i}$.

(vi) If $d_{i} \in D_{i}, p \in P$ and $p$ is presented prior to $d_{i}$ then $\mathrm{D}(p) \subseteq D_{1} \cup \ldots \cup D_{i-1}$.

\section{Proof}

(i) From $e_{i} \in \mathrm{U}\left(d_{i}\right)$ but $e_{i} \notin \mathrm{U}\left(d_{j}\right)$ for all $j>i$ and the linear order on the up-sets of elements we obtain $\mathrm{U}\left(d_{i}\right) \supsetneq \mathrm{U}\left(d_{j}\right)$.

(ii) The last significant point $e_{m-1}$ has the largest down set in $\mathbf{P}$. This means that all points outside $\mathrm{D}\left(e_{m-1}\right)$, namely $D_{m}=P-\mathrm{D}\left(e_{m-1}\right)$, have empty up sets.

(iii) This follows from (ii) and the fact that $D_{i}$ is the subset of maximal points of $\mathbf{P}_{e_{i}}$. 
(iv) Suppose $i>1$ since for $i=1$ the claim is obvious. Clearly, $d_{i} \nless e_{i-1}$. But if $d_{i}>e_{i-1}$ then $\mathrm{D}\left(e_{i-1}\right) \subsetneq \mathrm{D}\left(d_{i}\right)$. Therefore $d_{i}$ is the next significant point after $e_{i-1}$ or $e_{i}$ was presented before $d_{i}$. Both cases are impossible as $D_{i} \subseteq \mathrm{D}\left(e_{i}\right)$, thus $d_{i} \| e_{i-1}$. Now, if $d_{i}<p$ then by the linearity of down sets we obtain $D_{1} \cup \ldots \cup$ $D_{i-1}=\mathrm{D}\left(e_{i-1}\right) \subsetneq \mathrm{D}(p)$.

(v) Suppose that $p>d$ for some $d \in D_{j}, j>i$. Then (iv) guarantees that $D_{i} \subseteq$ $\mathrm{D}(p)$. This implies $d_{i}<p$, contradicting the assumptions.

(vi) If $i=m$ then the thesis is trivial as $D_{m}$ is the set of maximal points of $\mathbf{P}$. Thus, suppose $i<m$. Recall that $e_{i}$ is the first presented point which dominates every point from $D_{i}$. Now as $p$ is presented prior to $d_{i}$ and $d_{i}$ precedes $e_{j}$ for $j \geqslant i$, we get that $p$ cannot dominate any point from $D_{j}$.

With the next fact we prove that ALG always chooses a chain whose top is in the highest of the layers which contain tops of valid chains.

Fact 2.8 Let $_{i} \in D_{i}, d_{j} \in D_{j}, i<j$ and $d_{i}, d_{j}<x$. If $d_{i}$ is an ALG-top in $\mathbf{P}_{x}$ and ALG uses its chain on $x$ then $d_{j}$ is not an ALG-top in $\mathbf{P}_{x}$.

Proof At the moment when Spoiler presents $x$, both $d_{i}$ and $d_{j}$ are already introduced (P is up-growing). Since $e_{j-1}$ was presented before $d_{j}, d_{i} \in \mathrm{D}\left(e_{j-1}\right)$ and $d_{j} \nless e_{j-1}$ we conclude that $\mathrm{U}\left(d_{i}\right) \supsetneq \mathrm{U}\left(d_{j}\right)$ in $\mathbf{P}_{x}$. Hence if both $d_{i}$ and $d_{j}$ are valid tops for $x$ then ALG has a preference for using the chain of $d_{j}$ for $x$.

Before we proceed with the proof we introduce the concept of a predecessor and a successor of a point with respect to some fixed chain partition. Let $\mathcal{C}$ be the chain partition of $\mathbf{P}=(P, \leqslant)$. For $p \in P$ with $p \in C$ for a chain $C \in \mathcal{C}$ we define:

(i) The predecessor of $p$ in $\mathcal{C}$ is the point preceding $p$ in $C$ (if $p$ is the least point in $C$ then the predecessor of $p$ does not exist).

(ii) The successor of $p$ in $\mathcal{C}$ is the point succeeding $p$ in $C$ (if $p$ is the largest point in $C$ then the successor of $p$ does not exist).

We fix an optimal chain partition $\mathcal{O}$ of $\mathbf{P}$. Since width $(\mathbf{P}) \leqslant w$ this partition consists of at most $w$ chains. With respect to this partition we denote the predecessor and the successor of $p \in P$ by $o^{-}(p)$ and $o^{+}(p)$, respectively. Analogously, we refer to the predecessor and the successor of $p$ with respect to the the chain partition constructed by ALG as alg- $(p)$ and $\operatorname{alg}^{+}(p)$.

We arrive at the key concept of the proof: the alternating paths. Each such path starts at the bottom of an ALG-chain. We propose to understand it as a chain of events originating from the starting bottom. By counting the number of such paths we will get a bound on the number of chains used by ALG.

For each ALG-chain $\alpha$ define an alternating path $q=\left(q_{0}, \ldots\right)$ as follows:

(i) $q_{0}$ is the bottom point of $\alpha$,

(ii) $q_{2 i+1}=o^{-}\left(q_{2 i}\right)$, if $o^{-}\left(q_{2 i}\right)$ does exist,

(iii) $q_{2 i+2}=\operatorname{alg}^{+}\left(q_{2 i+1}\right)$, if $\operatorname{alg}^{+}\left(q_{2 i+1}\right)$ does exist.

We claim that for each path $q$ all the $q_{2 i}$ 's are pairwise distinct and so are all the $q_{2 i+1}$ 's. Indeed, note that $q_{0} \neq q_{2 i}$ for $i>0$ as $q_{2 i}=\operatorname{alg}^{+}\left(q_{2 i-1}\right)$ is not a bottom of an ALG-chain, while $q_{0}$ is defined as a bottom. Now suppose that the claim does 
not hold and consider the least $i$ such that $q_{i}=q_{j}$ for some $j>i$ where $i$ and $j$ have the same parity. If $i$ and $j$ are even we get $q_{i-1}=\operatorname{alg}^{-}\left(q_{i}\right)=\operatorname{alg}^{-}\left(q_{j}\right)=q_{j-1}$ and if they are odd we get $q_{i-1}=o^{+}\left(q_{i}\right)=o^{+}\left(q_{j}\right)=q_{j-1}$. In both cases this contradicts the choice of $i$. This fact implies that the alternating paths are finite. Note that an alternating path $q=\left(q_{0}, \ldots, q_{l}\right)$ is uniquely determined by any $q_{i} \in q$ together with the information whether $q_{i}$ is an odd or a even element. Altogether we have proven the following fact:

Fact 2.9 For an alternating path $q=\left(q_{0}, \ldots, q_{l}\right)$ all the $q_{i}$ 's with the same parity of indices are pairwise distinct, i.e. $q_{2 i} \neq q_{2 j}$ and $q_{2 i+1} \neq q_{2 j+1}$ for $i \neq j$. Moreover, each $p \in P$ occurs in at most two alternating paths: once with an odd index and once with an even index.

For an alternating path $q=\left(q_{0}, \ldots, q_{l}\right)$ we call the $q_{2 i}$ 's the up-points of $q$ and the $q_{2 i+1}$ 's the down-points of $q$. An alternating path $q=\left(q_{0}, \ldots, q_{l}\right)$ is an up-path if its last point is an up-point, otherwise, $q$ is a down-path. Note that an up-path connects the bottom of an ALG-chain with the bottom of an $\mathcal{O}$-chain, hence, there are at most $w$ up-paths (see Fact 2.12). The goal is to bound the number of down-paths.

From our perspective the important layers in the partition of $P=D_{1} \cup \ldots \cup D_{m}$ will be those containing at least one end point of a down-path. Define

$$
I=\left\{i \text { : there is a down-path ending in } D_{i}\right\}=\left\{i_{0}<i_{1}<\ldots<i_{s}\right\} .
$$

Note that $m \notin I$ being an end point of a down-path has a non-empty up set, while up sets of all points in $D_{m}$ are empty (see Fact 2.7(ii)). This means

$$
i_{s}<m \text {. }
$$

From the definition of $I$ we immediately obtain:

Fact 2.10 There is an ALG-top in every $D_{i_{j}}$, for $0 \leqslant j \leqslant s$.

The next fact basically states that $D_{i_{0}} \cup \ldots \cup D_{m}$ induce an order of height at most 3 .

Fact 2.11 $D_{i_{0}+1} \cup \ldots \cup D_{m-1}$ is an antichain.

Proof In order to get a contradiction suppose that there are $d, d^{\prime} \in D_{i_{0}+1} \cup \ldots \cup D_{m-1}$ and that $d<d^{\prime}$. As $d^{\prime}$ dominates a point from a layer higher than $D_{i_{0}}$, we get from Fact 2.7(iv) that $D_{i_{0}} \subseteq \mathrm{D}\left(d^{\prime}\right)$. On the other hand, since $d^{\prime} \notin D_{m}$ there must be some point $d^{\prime \prime}>d^{\prime}$ (see Fact 2.7(ii)). Fix some $t \in D_{i_{0}}$ that remains an ALG-top throughout the game (such $t$ exists by Fact 2.10). Recall that $p_{n}$, the last point presented by Spoiler, is incomparable with $t$ as otherwise $p_{n}$ would not be assigned to a new ALGchain. Together this shows that $p_{n}$ and $t<d^{\prime}<d^{\prime \prime}$ form a $(\mathbf{3}+\mathbf{1})$-configuration. This is impossible since $\mathbf{P}$ is a semi-order.

We now introduce variables that count the number of paths with respect to their end points. Define

$x_{U}$ the total number of up-paths, 
$x_{j} \quad$ the number of down-paths ending in $\bigcup_{i \geqslant i_{j}} D_{i}$, for $j=0, \ldots, s$, $x_{s+1}=0$.

In terms of these variables the number of chains used by ALG can be expressed as

$$
x_{U}+x_{0}
$$

Fact 2.12 $x_{U} \leqslant w$.

Proof Consider the set $U$ of end points of up-paths. By Fact 2.9 these end points are pairwise distinct and $|U|=x_{U}$. As each point in $U$ is a bottom point of its chain in $\mathcal{O}$ these points belong to different chains in $\mathcal{O}$. Therefore, $x_{U}=|U| \leqslant|\mathcal{O}| \leqslant w$.

Throughout the rest of the paper our efforts are targeted on bounding the number $x_{0}$ of down-paths. In the following two lemmas we will present a system of inequalities involving $x_{0}$ and the other $x_{i}$ 's. From these inequalities we will derive the desired constraints on the value of $x_{0}$.

Lemma $2.13 x_{0}+x_{0}-x_{1} \leqslant w$.

Lemma $2.14 x_{0}+x_{1}+\ldots+x_{j}+\left(x_{j}-x_{j+1}\right) \leqslant w$, for $j=1, \ldots, s$.

We need some preparations for the proofs of the lemmas. In the counting arguments on which the proofs are based we will be often showing that certain sets are disjoint. Below we introduce a criterion that will help us do the bookkeeping.

Point $p \in P$ is a good point if $o^{-}(p)$ exists and $o^{-}(p)$ is an ALG-top at the moment when $p$ is presented, i.e., $o^{-}(p)$ is a top of its ALG-chain in $\mathbf{P}_{p}$. Simple enough, point $p$ is considered bad if it is not good.

Fact 2.15 states that the penultimate point of a down-path is always good. Fact 2.16 says that if a down-path ends in $D_{i_{j}}$ then it contains $j+1$ bad up-points. Fact 2.17 is a technical statement used in the proof of Lemma 2.14.

Fact 2.15 The penultimate point of a down-path is a good point and lies in $D_{m}$.

Proof Let $q=\left(q_{0}, \ldots, q_{l-1}, q_{l}\right)$ be a down-path. We have $q_{l}=o^{-}\left(q_{l-1}\right)$ and since $q$ ends with $q_{l}=o^{-}\left(q_{l-1}\right)$ this point must be an ALG-top in P. This shows that $q_{l-1}$ is good.

To prove that $q_{l-1} \in D_{m}$ we are going to prove an equivalent condition that the up set of $q_{l-1}$ is empty (see Fact 2.7(ii)). Recall that the last point presented in $\mathbf{P}$, namely $p_{n}$, receives a new ALG-chain. This means that there is no valid chain for $p_{n}$ and in particular, $p_{n} \ngtr q_{l}$. From $\mathrm{U}\left(p_{n}\right)=\emptyset$ we get $p_{n} \| q_{l}$. Now, if there was a point $x>q_{l-1}$ then $p_{n}, q_{l}, q_{l-1}, x$ would form a $(\mathbf{3}+\mathbf{1})$-configuration. This is impossible since $\mathbf{P}$ is a semi-order.

Fact 2.16 A down-path $q$ ending in $D_{i_{j}}$ contains $j+1$ bad up-points $\left\{y_{0}, \ldots, y_{j}\right\}$ such that $o^{-}\left(y_{k}\right) \in D_{i_{k}}$, for $0 \leqslant k \leqslant j$. 
Proof Fix $k$ and define $y_{k}$ to be the first up-point in $q$ such that $o^{-}\left(y_{k}\right) \in D_{l}$ for some $l \geqslant i_{k}$. Such point does exist, as the penultimate point in $q$ is a candidate for $y_{k}$.

Claim $y_{k}$ is a bad point.

Suppose $y_{k}$ is the first point of $q$. In this case ALG uses a new chain on $y_{k}$. Since ALG is greedy there is no valid chain for $y_{k}$ at the moment it is presented. In particular, $o^{-}\left(y_{k}\right)$ is not an ALG-top in $\mathbf{P}_{y_{k}}$ and therefore $y_{k}$ is a bad point.

If $y_{k}$ is not the first point of $q=\left(\ldots, p, o^{-}(p), y_{k}, o^{-}\left(y_{k}\right), \ldots\right)$, then we know that $p$ did not qualify for $y_{k}$ and therefore $\operatorname{alg}^{-}\left(y_{k}\right)=o^{-}(p) \in D_{1} \cup \ldots \cup D_{i_{k}-1}$. From Fact 2.8 it follows that $o^{-}\left(y_{k}\right)$ is not an ALG-top in $\mathbf{P}_{i_{k}}$ and again $y_{k}$ is bad. This proves the claim.

It remains to show that $o^{-}\left(y_{k}\right) \in D_{i_{k}}$. Fix an ALG-top $t \in D_{i_{k}}$ (Fact 2.10). Note that $t \nless y_{k}$ as otherwise ALG would have given preference to the chain of $t$ instead of the one it used for $y_{k}$. From Fact 2.7(v) we get $\mathrm{D}\left(y_{k}\right) \subseteq D_{1} \cup \ldots \cup D_{i_{k}}$. From this and the definition of $y_{k}$ we conclude $o^{-}\left(y_{k}\right) \in D_{i_{k}}$.

Fact 2.17 For every $j(0 \leqslant j \leqslant s)$ and down-path q there is an up-point $u \in q$ such that one of the following two conditions is true:

(i) $u \in D_{i_{j}+1} \cup \ldots \cup D_{m}$ and $o^{-}(u) \in D_{i_{0}}$, or

(ii) $u$ is good and $o^{-}(u) \in D_{i_{0}+1} \cup \ldots \cup D_{i_{j}-1}$.

Proof Fix $j$ and a down-path $q$. Let $a$ be the last up-point in $q$ such that $o^{-}(a) \in D_{k}$ for some $k \leqslant i_{0}$. There is such a point as by Fact 2.16 each down-path has an up-point $y$ with $o^{-}(y) \in D_{i_{0}}$.

First we are going to prove that $o^{-}(a) \in D_{i_{0}}$. It is trivial if $o^{-}(a)$ is the last point of $q$ as the first layer with an end of a down-path is $D_{i_{0}}$. If $o^{-}(a)$ is not the last point then let $b$ be the next point of $q$, i.e. $q=\left(\ldots, a, o^{-}(a), b, o^{-}(b), \ldots\right)$. From the way point $a$ is chosen we have $o^{-}(b) \in D_{l}$, for some $l>i_{0}$. By Fact 2.7(iv) $b>o^{-}(b) \in$ $D_{l}$ implies $D_{i_{0}} \subseteq \mathrm{D}(b)$. Now, recall that there is an ALG-top $t \in D_{i_{0}}$ (Fact 2.10). In particular, at the moment when $b$ was introduced the chain of $t \in D_{i_{0}}$ was valid for $b$. Since ALG has chosen $\operatorname{alg}^{-}(b)=o^{-}(a) \in D_{k}$ we get $k \geqslant i_{0}$ (Fact 2.8). Since $a$ was chosen such that $o^{-}(a) \in D_{k}$ for some $k \leqslant i_{0}$ we get $k=i_{0}$.

If $a \in D_{i_{j}+1} \cup \ldots \cup D_{m}$ then $a$ fulfills the condition for the $u$ of (i) and we are done. From now on we deal with the case $a \in D_{k}$ for some $k \leqslant i_{j}$. As $a>o^{-}(a) \in D_{i_{0}}$ point $a$ must be somewhere in $D_{i_{0}+1} \cup \ldots \cup D_{m}$. We therefore know that

$$
a \in D_{i_{0}+1} \cup \ldots \cup D_{i_{j}} .
$$

Since $i_{j}<m$ (see Eq. 2) we have $a \notin D_{m}$, hence, there is a point $a^{\prime} \in P$ with $a^{\prime}>a$. Note that $o^{-}(a)$ can't be the last point of $q$. Otherwise, $a$ would be the penultimate point of $q$ which is in $D_{m}$ (Fact 2.15). Let $b$ be the successor of $o^{-}(a)$ in $q$, i.e. $q=$ $\left(\ldots, a, o^{-}(a), b, o^{-}(b), \ldots\right)$. We claim that Spoiler presents $b$ prior to $a$ in $\mathbf{P}$, i.e. $b \in \mathbf{P}_{a}$. From the definition of $a$ it follows that $b>o^{-}(b) \in D_{l}$, for some $l>i_{0}$ and hence $D_{i_{0}} \subseteq \mathrm{D}(b)$. (Fact 2.7(v)). Consider an ALG-top $t \in D_{i_{0}}$ (Fact 2.10) and recall that the last point $p_{n}$ presented in $\mathbf{P}$ is put into a new ALG-chain and therefore $p_{n} \| t$. Now, if $a>t$ then $p_{n}$ together with $t<a<a^{\prime}$ would form a $(\mathbf{3}+\mathbf{1})$-configuration. Therefore $a \| t$ while obviously $a>o^{-}(a)$. Suppose that $a$ is presented by Spoiler prior to $b$. This implies that at the moment when $b$ is introduced (i.e. in $\mathbf{P}_{b}$ ) we have 
$\mathrm{U}(t) \subsetneq \mathrm{U}\left(o^{-}(a)\right)$ and therefore ALG would prefer the chain of $t$ over the chain of $\operatorname{alg}^{-}(b)=o^{-}(a)$ to be used for $b$. With this contradiction we have proved the claim that the order of presentation is $P=(\ldots, b, \ldots, a, \ldots)$.

Among all up-points in $q$ that Spoiler presents before $a$, let $c$ be the closest to the end of $q$. There is such a point as $b$ is an up-point of $q$ and it is presented prior to $a$. Since $b$ comes after $a$ on $q$ this also holds for $c$, i.e.,

$$
q=\left(\ldots, a, o^{-}(a), \ldots, c, o^{-}(c), \ldots\right)
$$

The last step in the proof is to show that $c$ fulfills the condition for the $u$ of (ii).

First, we show that $c$ is good. If not, $z=\operatorname{alg}^{+}\left(o^{-}(c)\right)$ would be defined and had to be presented prior to $c$ (since $\mathbf{P}$ is up-growing), so before $a$ as well. But then $z$ would be also an up-point of $q$ which contradicts the choice of $c$.

It remains to prove that $o^{-}(c) \in D_{i_{0}+1} \cup \ldots \cup D_{i_{j}-1}$. Recall that $a$ is the last uppoint of $q$ with $o^{-}(a) \in D_{k}$ for $k \leqslant i_{0}$. Since $c$ comes later than $a$ on $q$ (see Eq. 4) we have $o^{-}(c) \in D_{k}$ for some $k \geqslant i_{0}+1$. To bound $k$ from above recall that $a \in D_{i_{0}+1} \cup$ $\ldots \cup D_{i_{j}}$ and $o^{-}(c)$ is presented prior to $a$. Applying this to Fact 2.7.(vi) we get that $o^{-}(c) \in \bigcup_{i<i_{j}} D_{i}$. This finishes the proof of Fact 2.17.

With these preparations we are ready for the proofs of Lemmas 2.13 and 2.14.

Proof of Lemma 2.13 We are going to construct an antichain in $\mathbf{P}$ of size $x_{0}+x_{0}-x_{1}$. This implies the statement.

By Fact 2.16 a down-path $q$ contains a bad up-point $y_{q}$ with $o^{-}\left(y_{q}\right) \in D_{i_{0}}$. Collect these points in a set $Y=\left\{y_{q}: q\right.$ is a down-path $\}$. Since $x_{0}$ is just the number of down-paths and $Y$ contains a point from each down-path we get $|Y|=x_{0}$. Let $Z$ be the set of penultimate points of down-paths ending in $D_{i_{0}}$. The number of such paths is $x_{0}-x_{1}$. hence $|Z|=x_{0}-x_{1}$. From Fact 2.15 we know that points in $Z$ are good. Summarizing:

(i) $|Y|=x_{0}$, all $y \in Y$ are bad and satisfy $o^{-}(y) \in D_{i_{0}}$.

(ii) $|Z|=x_{0}-x_{1}$, all $z \in Z$ are good and satisfy $o^{-}(z) \in D_{i_{0}}$.

This implies that $Y$ and $Z$ are disjoint and

$$
x_{0}+x_{0}-x_{1}=|Y|+|Z|=|Y \cup Z|=\left|o^{-}(Y \cup Z)\right| \leqslant\left|D_{i_{0}}\right| \leqslant w,
$$

where the last inequality holds as $D_{i_{0}}$ is an antichain in $\mathbf{P}$ (Fact 2.7(iii)).

Proof of Lemma 2.14 The basic idea of the proof is similar to the proof Lemma 2.13 but the details are more involved. For fixed $j$ we construct a set consisting of $x_{0}+$ $x_{1}+\ldots+x_{j}+x_{j}-x_{j+1}$ points such that the points of the set belong to different chains in $\mathcal{O}$. As $\mathcal{O}$ contains at most $w$ chains, this implies the inequality with index $j$.

Fix $j$. First we construct a set of size $x_{1}+\ldots+x_{j}$. For a down-path $q$ ending in $D_{i_{k}}$ put $r(q)=\min (k, j)$. By Fact 2.16 each $q$ contains a set $Y_{q}$ of $r(q)$ bad up-points $y$ with $o^{-}(y) \in D_{i_{1}} \cup \ldots \cup D_{i_{j}}$. Let $Y$ be the union of all these sets $Y_{q}$. We claim that that all the $Y_{q}$ 's are pairwise disjoint. This is true because no point occurs in more 
than one alternating path as an up-point (Fact 2.9). The claim implies $|Y|=\sum_{q}\left|Y_{q}\right|$. We determine the size of $Y$ as follows

$$
\begin{aligned}
|Y| & =\sum_{k=1}^{s} \sum_{\substack{q \text { ending } \\
\text { in } D_{i_{k}}}}\left|Y_{q}\right|=\sum_{k=1}^{s} \sum_{\substack{q \text { ending } \\
\text { in } i_{i_{k}}}} r(q)=\sum_{k=1}^{s} \min (k, j) \cdot\left(x_{k}-x_{k+1}\right) \\
& =\sum_{k=1}^{j} k \cdot\left(x_{k}-x_{k+1}\right)+\sum_{k=j+1}^{s} j \cdot\left(x_{k}-x_{k+1}\right)=x_{1}+\ldots+x_{j}-j \cdot x_{s+1} \\
& =x_{1}+\ldots+x_{j} .
\end{aligned}
$$

For further reference we collect the important properties of $Y$ :

(i) $|Y|=x_{1}+\ldots+x_{j}$.

(ii) All $y \in Y$ are bad and $o^{-}(y) \in D_{i_{1}} \cup \ldots \cup D_{i_{j}} \subseteq D_{i_{0}+1} \cup \ldots \cup D_{i_{j}}$.

The second set to consider is:

$$
Z=\left\{z: \text { there is a down-path } q=\left(\ldots, z, o^{-}(z)\right) \text { and } o^{-}(z) \in D_{i_{j}}\right\}
$$

This is the set of the penultimate points of down-paths ending in $D_{i_{j}}$. The penultimate points of down-paths are up-points and hence all distinct (Fact 2.9). From Fact 2.15 we know that all points in $Z$ are good. Summarizing:

(i) $|Z|=x_{j}-x_{j+1}$,

(ii) All $z \in Z$ are good and $o^{-}(z) \in D_{i_{j}}$

With a help of Fact 2.17 we construct a third set $U$. Each down-path $q$ contains an up-point $u_{q}$ satisfying property (i) or (ii) of Fact 2.17. The set $U$ is the collection of all these points. Since no point is an up-point of more than one path all the $u_{q}$ 's are distinct. Since there are $x_{0}$ down-paths we have $|U|=x_{0}$. We partition the set $U=\left\{u_{q}: q\right.$ down-path $\}$ into three parts $U_{1}, U_{2}$ and $U_{3}$ as follows:

(i) $U_{1}$ is the set of $u \in U$ with $u \in D_{i_{j}+1} \cup \ldots \cup D_{m-1}$ and $o^{-}(u) \in D_{i_{0}}$.

(ii) $U_{2}$ is the set of $u \in U$ with $u \in D_{m}$ and $o^{-}(u) \in D_{i_{0}}$.

(iii) $U_{3}$ is the set of $u \in U$ such that $u$ is good and $o^{-}(u) \in D_{i_{0}+1} \cup \ldots \cup D_{i_{j-1}}$.

The following properties of sets $Y, Z$ and $U_{1}, U_{2}, U_{3}$ are crucial:

(1) The sum of sizes of $U_{1}, U_{2}, U_{3}, Y$ and $Z$ is $x_{0}+x_{1}+\ldots+x_{j}+\left(x_{j}-x_{j+1}\right)$.

(2) $U_{3}, Y$ and $Z$ are disjoint. Indeed points in $Y$ are bad while points in $Z$ and $U_{3}$ are good. The predecessors in $\mathcal{O}$-chains show that $Z$ and $U_{3}$ are disjoint.

(3) $o^{-}\left(U_{3} \cup Y \cup Z\right) \subseteq D_{i_{0}+1} \cup \ldots \cup D_{i_{j}}$ and $U_{1} \subseteq D_{i_{j+1}} \cup \ldots \cup D_{m-1}$.

(4) Points in $U_{2} \cup D_{i_{0}+1} \cup \ldots \cup D_{m-1}$ lie in different $\mathcal{O}$-chains. Indeed, $D_{i_{0}+1} \cup \ldots \cup$ $D_{m-1}$ is an antichain (Fact 2.11) and $\mathcal{O}$-chains of points from $U_{2}$ skip all layers between $D_{i_{0}}$ and $D_{m}$, i.e., they avoid $D_{i_{0}+1} \cup \ldots \cup D_{m-1}$. 
We are now ready to complete the proof:

$$
\begin{aligned}
x_{0}+\left(x_{1}+\ldots+x_{j}\right)+\left(x_{j}\right. & \left.-x_{j+1}\right)= \\
{ }^{(1)} & =\left(\left|U_{1}\right|+\left|U_{2}\right|+\left|U_{3}\right|\right)+|Y|+|Z| \\
{ }^{(2)} & =\left|U_{2}\right|+\left|U_{3} \cup Y \cup Z\right|+\left|U_{1}\right| \\
& =\left|U_{2}\right|+\left|o^{-}\left(U_{3} \cup Y \cup Z\right)\right|+\left|U_{1}\right| \\
{ }^{(3)} & \leqslant\left|U_{2}\right|+\left|D_{i_{0}+1} \cup \ldots \cup D_{m-1}\right| \\
{ }^{(4)} & \leqslant|\mathcal{O}| \\
& \leqslant w .
\end{aligned}
$$

Lemmas 2.13 and 2.14 provide us with a system of inequalities involving all the $x_{i}$ 's:

$$
x_{0}+x_{1}+\ldots+x_{j}+x_{j}-x_{j+1} \leqslant w, j=0, \ldots, k .
$$

Note that these are the inequalities used for the lower bound. Here, for the completion of the proof of Proposition 2.6 we need a final lemma to bound the number $x_{0}+w$ of chains used by Algorithm.

Lemma 2.18 $x_{0} \leqslant(\varphi-1) \cdot w$.

Proof We weight the $j$ th inequality with the Fibonacci number $F_{2(k-j)+1}$ and take the sum of weighted inequalities:

$$
\sum_{j=0}^{k} \sum_{i=0}^{j} x_{i} F_{2(k-j)+1}+\sum_{j=0}^{k}\left(x_{j}-x_{j+1}\right) F_{2(k-j)+1} \leqslant w \sum_{j=0}^{k} F_{2(k-j)+1} .
$$

Using the well-known Fibonacci identity $\sum_{j=0}^{k} F_{2(k-j)+1}=\sum_{j=0}^{k} F_{2 j+1}=F_{2 k+2}$ we can simplify the double-sum:

$$
\sum_{j=0}^{k} \sum_{i=0}^{j} x_{i} F_{2(k-j)+1}=\sum_{i=0}^{k} x_{i} \sum_{j=i}^{k} F_{2(k-j)+1}=\sum_{j=0}^{k} x_{j} F_{2(k-j)+2} .
$$

This and again using the Fibonacci identity on the right hand side allows us to rewrite inequality 5 :

$$
\sum_{j=0}^{k} x_{j} F_{2(k-j)+2}+\sum_{j=0}^{k} x_{j} F_{2(k-j)+1}-\sum_{j=1}^{k+1} x_{j} F_{2(k-j)+3} \leqslant w F_{2 k+2} .
$$

Using the Fibonacci recursion and the fact that $x_{k+1}=0$ this reduces to

$$
x_{0} F_{2 k+2}+x_{0} F_{2 k+1} \leqslant w F_{2 k+2} .
$$


This in turn can be rewritten into

$$
x_{0} \leqslant \frac{F_{2 k+2}}{F_{2 k+3}} \cdot w \leqslant(\varphi-1) \cdot w .
$$

The last inequality is due to the fact that the sequence $\left(\frac{F_{2 k+2}}{F_{2 k+3}}\right)_{k \geqslant 0}$ is monotonically increasing with the limit $\varphi-1$.

The statement of Lemma 2.18 was the last piece of the puzzle. The number of chains used by ALG is bounded by

$$
x_{U}+x_{0} \leqslant w+(\varphi-1) \cdot w .
$$

This completes the proof of Proposition 2.6 and hence of Theorem 1.1.

Open Access This article is distributed under the terms of the Creative Commons Attribution Noncommercial License which permits any noncommercial use, distribution, and reproduction in any medium, provided the original author(s) and source are credited.

\section{References}

1. Baier, P., Bosek, B., Micek, P.: On-line chain partitioning of up-growing interval orders. Order 24(1), 1-13 (2007)

2. Bosek, B., Felsner, S., Kloch, K., Krawczyk, T., Matecki, G., Micek, P.: On-line chain partitions of orders: a survey. Order (2011). doi:10.1007/s11083-011-9197-1

3. Felsner, S.: On-line chain partitions of orders. Theor. Comput. Sci. 175(2), 283-292 (1997)

4. Kierstead, H.A.: An effective version of Dilworth's theorem. Trans. Am. Math. Soc. 268(1), 63-77 (1981)

5. Kierstead, H.A.: Recursive ordered sets. In: Combinatorics and Ordered Sets, Arcata, Calif., 1985. Contemp. Math., vol. 57, pp. 75-102. Amer. Math. Soc., Providence, RI (1986)

6. Kierstead, H.A., Trotter, Jr., W.T.: An extremal problem in recursive combinatorics. In: Proceedings of the Twelfth Southeastern Conference on Combinatorics, Graph Theory and Computing, Vol. II, (Baton Rouge, La., 1981), vol. 33, pp. 143-153 (1981)

7. Möhring, R.H.: Computationally tractable classes of ordered sets. In: Algorithms and Order, pp. 105-193. Kluwer Acad. Publ., Dordrecht (1989) 